\title{
An optimization framework for tracking droplets in fire water spray images
}

\author{
Joachim Lundberg $^{1} \quad$ Marius Lysaker ${ }^{1}$ \\ ${ }^{1}$ Faculity of Technology, Telemark University College, Norway, joachim. lundberg@ hit.no
}

\begin{abstract}
The properties of the flow from fire water nozzles, like droplet size and velocity distribution within the spray, are known to influence the fire suppression efficiency. To analyze the flow properties, the water spray is recorded with the use of a high-speed camera and laser light. Typically, each image of the water spray may contain tens of droplets, yielding a huge number of possible droplet paths between adjacent frames, i.e. $n$ droplets in two subsequent frames generates $n^{2}$ possible droplet paths with $n$ ! possible configurations using brute-force approach. In this paper, we propose an optimization method based on the Hungarian algorithm to calculate the droplet paths. Using this framework, each droplet path is optimized with respect to droplet position, droplet size and droplet velocity.
\end{abstract}

Keywords: Droplets, Image Processing, Tracking

\section{Introduction}

The Norwegian petroleum industry has developed a standard for the technical safety of offshore installations (NORSOK S-001, 2008). When dimensioning accidental load with this standard, the deluge or fire water spray may be considered as a risk reducing measure for equipment and pipes, but not for the structural elements or fire partition (NORSOK S-001, 2008). In risk evaluation, proper documentation of the suppression effect and reliability has to be provided when water is used as a fire risk reduction measure. The standard states that the deluge system shall be automatically activated upon confirmed gas detection when it is used for explosion mitigation.

In the literature, the availability of data on fire water spray is limited. Often the spray is described only by the orifice diameter of the fire water nozzles and spray angle. However, the flow properties of the spray (i.e., size and velocity distribution of the droplets) are known to influence the suppression efficiency. Small droplets will follow the gas flow, evaporate quickly, and cool the fire gases. In contrast, large droplets have high momentum and are more likely to reach the source of the fire and to cool objects such as process equipment and pipes.

In the 90's, Jackman (1992) manually measured the droplet sizes and velocities for some commercial nozzles, using an image-based method to determine size from photographic high-speed film images. Sheppard (2002) found the velocity field and droplet sizes using particle image velocimetry (PIV) and phase Doppler anemometry (PDA). PDA is a common technique for characterizing droplet distributions in sprays. In PDA measurements the droplets scatter the laser light at an angle that can be used to determine the droplet diameter. The light scattering is actually a measure of the curvature of the surface of the droplet; however, this technique has limitations with non-spherical droplets (Kashdan et al., 2007). Non-spherical droplets may be excluded by the data acquisition software, although this results in uncertainty in the PDA measurements. Recently, Zhou et al. (2012) used an image-based method to characterize the spray from a fire sprinkler in the near and far field of the sprinkler tip, with the near field measurements providing input that could be used in numerical fire models.

Image processing techniques are currently being used for analyzing sprays because they are relatively simple and economical compared to other methods like PDA (Koh, 2001). They also have advantages to other techniques regarding non-spherical droplets. However, it can be demanding to use these techniques due to the number of frames that need to be analyzed to get a statistically meaningful distribution (Lee \& Kim, 2004). In shadow-imaging techniques, the depth of field effect is one of the major contributions to measurement error. (Lee \& Kim, 2004).

To determine the droplet paths for each individual droplet of the spray, an optimization framework based on the Hungarian algorithm will be used. The overall objective is to obtain the spray characteristics of a medium velocity deluge nozzle used on an offshore installation in the North Sea, and make a spray characterizing method with an in-house image processing code.

Other post-processing codes for analyzing shadowimages have been tested. But for fire water spray, only commercial codes where the tracking algorithm is not presented are available.

\section{Experimental setup}

The experimental setup is located inside a container and consists of the following equipment: a laser with transmitting optics, a deluge nozzle, a high-speed 


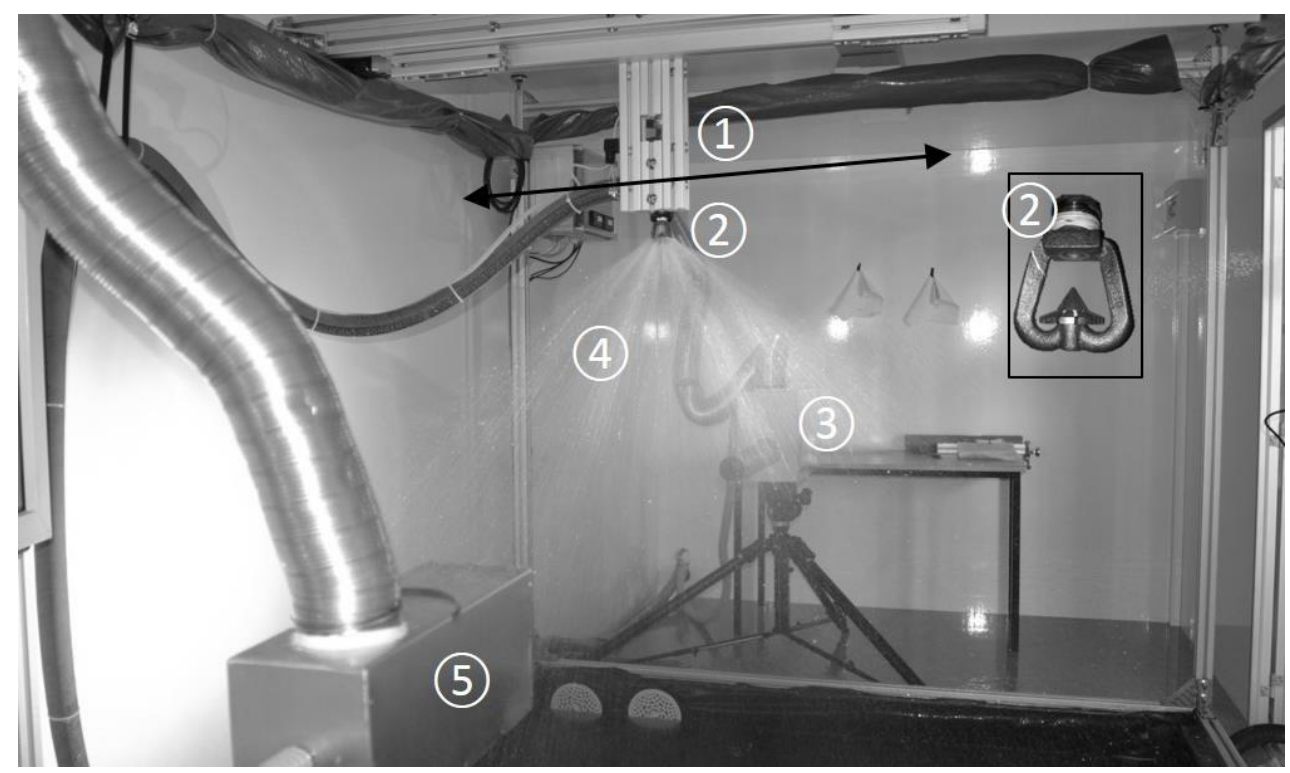

Figure 1. The experimental setup for fire water droplet measurement rig: 1) traverse with nozzle mounting, 2) deluge nozzle, 3) high-speed camera and long-distance microscope lens, 4) deluge spray, 5) laser transmitting optics.

camera, a long-distance microscope lens, a traverse with nozzle mounting and auxiliary equipment. The deluge nozzle is a Tyco MV34-110 medium velocity nozzle. According to the producer, the nozzle produces a coneshaped spray that is uniformly filled with medium velocity droplets. The sub image in Figure 1 shows a picture of the nozzle.

A high-speed camera is used to capture images of the spray illuminated with a laser as shown in Figure 2. The back illumination is a copper vapor laser (CVL) from Oxford Lasers. The laser generates short light pulses (25 $\mathrm{ns})$ with high effect $(20 \mathrm{~W})$ continually at $12.5 \mathrm{kHz}$. The camera and laser are synchronized to capture images at 12,500 frames per second. The high-speed camera is a Photron APX-RS monochrome camera. Two high magnification microscope lenses were used (Navitar 12 $\mathrm{X}$ with a focus range extender and Questar QM-1). The camera and lens have a narrow focus depth to produce sharp images of droplets in the focus plane with limited visibility of the droplets in front of or behind the plane. The technique is shown in Figure 2.

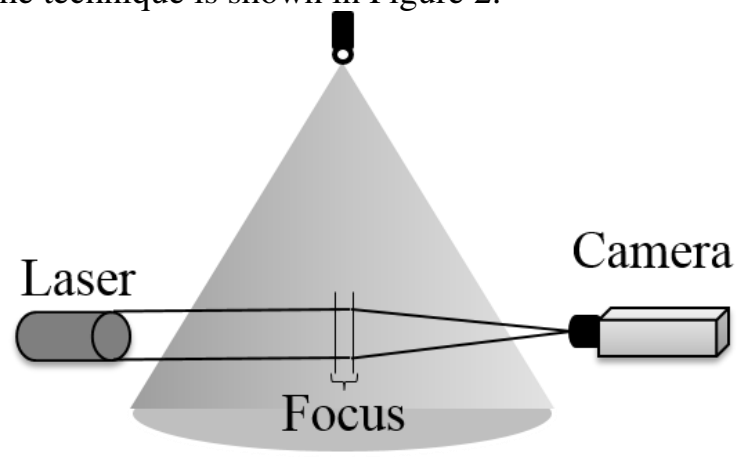

Figure 2. Experimental setup illustrating the narrow focus in the center plane of the spray.
The technique used in this work is called imagebased shadow-imaging. The camera will register the shadows of the droplets because the water droplets will scatter the light from the laser.

\section{Image processing}

The flow properties, like droplet size and velocity, generated with a fire water nozzle are known to vary within the spray (Sheppard, 2002). Therefore, to characterize the flow properties caused by a given fire water nozzle, hundreds of movies must be recorded. Each movie is recorded at a given location in the spray. Since each movie typically consists of 8,000-12,000 frames, millions of images need to be analyzed to obtain the velocity- and size- distribution for all locations of the fire water spray within a certain accuracy. Due to the number of images to be analyzed, an automatic postprocessing algorithm is required.

The post-processing algorithm is developed in MATLAB and uses MATLAB image processing toolbox, (Mathworks, 2009). Established and new image processing techniques are used to extract information about the fire water spray from the movies of the droplets. The size of the droplets can be determined by comparing the droplets in the movie to a calibration standard with a known size. The velocity can be found by comparing the position of the droplets in subsequent frames. By recording the measurements of several droplets, it is possible to find the droplet sizeand velocity distribution of the spray.

In this paper, we propose an algorithm that automatically calculates the size and velocity distribution in a given water spray movie. In short, the algorithm works as follows: 
1. Separate "in-focus" droplets from all "out-of-focus" droplets, and the background in each image.

2. Calculate the size and position of all "in-focus" droplets in each image.

3 . Find the velocity by comparing the position of the droplets in temporally adjacent frames.

\subsection{Segmentation}

The recorded images consist of droplets (both "infocus" and "out-of-focus" droplets), noise and the background. The background is the image without any droplets or noise. In an ideal image, the "in-focus" droplets are black, the "out-of-focus" droplets are gray and the background is white, meaning that it is easy to identify the "in-focus" droplets. However, in real measurements, due to varying illumination and optical phenomena, the background appears uneven and there is a smooth transition from "in-focus" to "out-of-focus" droplets, see Figure 3.

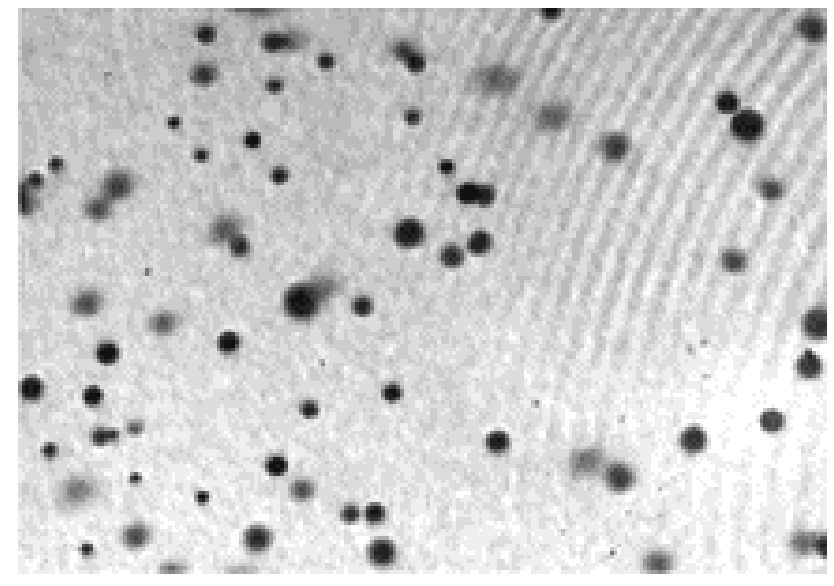

Figure 3. Original image from spray, $\boldsymbol{I}_{\boldsymbol{i}}$.

An estimate for the uneven background $B$ is found by averaging all frames within a movie, i.e.

$$
B=\frac{1}{n} \sum_{i=1}^{n} I_{i}
$$

where $I_{i}$ is the image at frame $i$ and $n$ is the total number of frames in the movie. Then, for each image in the movie, the background is subtracted:

$$
J_{i}=I_{i}-B, \quad \text { for } i=1: n .
$$

After the background subtraction routine, the images will mainly consist of noise and droplets, see Figure 4.

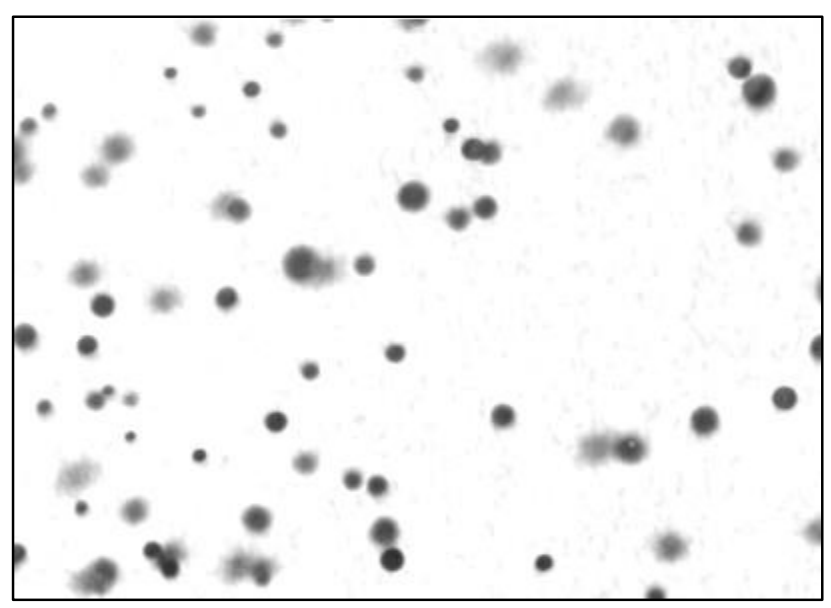

Figure 4. Image processed image $\boldsymbol{J}_{\boldsymbol{i}}$, where the background is removed from the original image.

The background $B$, which is subtracted from all of the images, is shown in Figure 5.

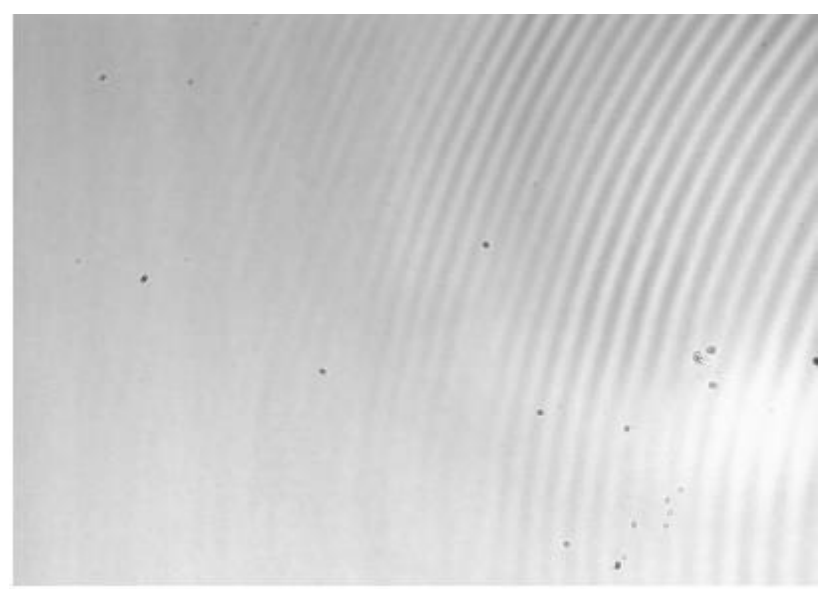

Figure 5. This figure shows the background found by (1).

The droplets can be isolated by applying a thresholding algorithm that divides the image into droplets and background (including noise). The Otsu (1979) algorithm is used to find the threshold value $t$. The optimal solution of $t$ maximizes the inter-class variance between the droplets and the background (Gonzales \& Woods, 2008). The threshold value is then used to divide the gray-scale image $J_{i}(x, y)$ into background and droplets, where $(x, y)$ represents the pixel coordinates. Every pixel value above the threshold $t$ is classified as background, and pixel value below $\mathrm{t}$ is identified as droplets, yielding the binary image

$$
T_{i}(x, y)=\left\{\begin{array}{ll}
0 & \text { if } J_{i}(x, y)<t \\
1 & \text { if } J_{i}(x, y) \geq t
\end{array} .\right.
$$

The threshold algorithm can be used both globally and locally, and thereby determine one global threshold value for an entire image, or one local threshold value for each defined area. The output of the threshold 
algorithm is a binary image, i.e. droplets and background, see Figure 6.

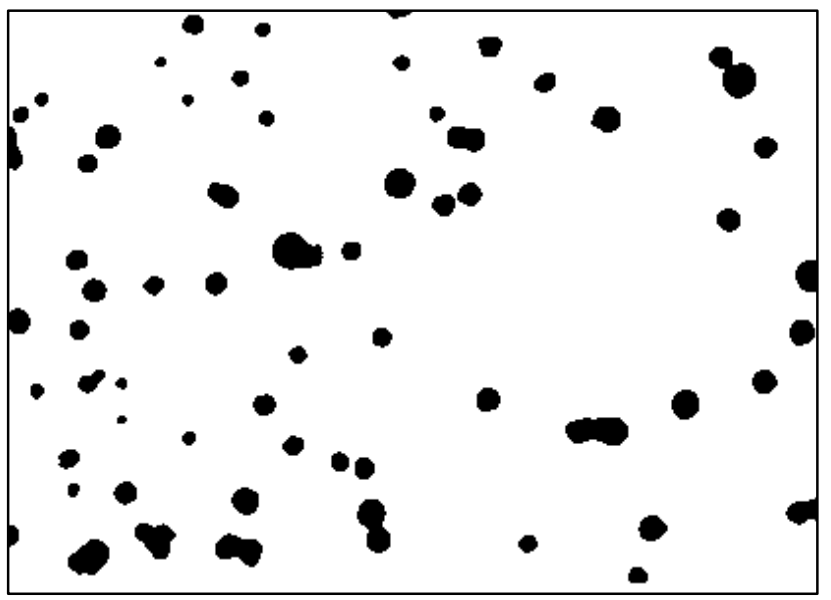

Figure 6. Thresholded image $\boldsymbol{T}_{\boldsymbol{i}}$ found with (3) showing droplets (black) and background (white).

\subsection{In-focus droplets}

The basic principle of shadow-imaging is that the camera will have a narrow depth of field that will make a sheet of droplets in focus while droplets in front of or behind this sheet will be blurred away. The thickness of the DOF sheet is dependent on the size and has to be calibrated. To qualify the "in-focus" droplets, the concept of gray-scale gradient at the boundary by Lecouna et al. (2000) is adopted. This method compares the gray-scale or intensity gradient at the boundary of the droplets to the intensity difference of the minimum of the droplet and the background.

Droplets in focus are counted to generate the spray distributions. In the cases examined here, the droplet sizes range from typically 100 to $1200 \mu \mathrm{m}$ and the velocity is between 1.0 and $15.0 \mathrm{~m} / \mathrm{s}$. These intervals will determine the range of droplets the image processing code is limited to find.

The qualified droplets are indexed from one to the number of recognized objects in the image as shown in Figure 7.

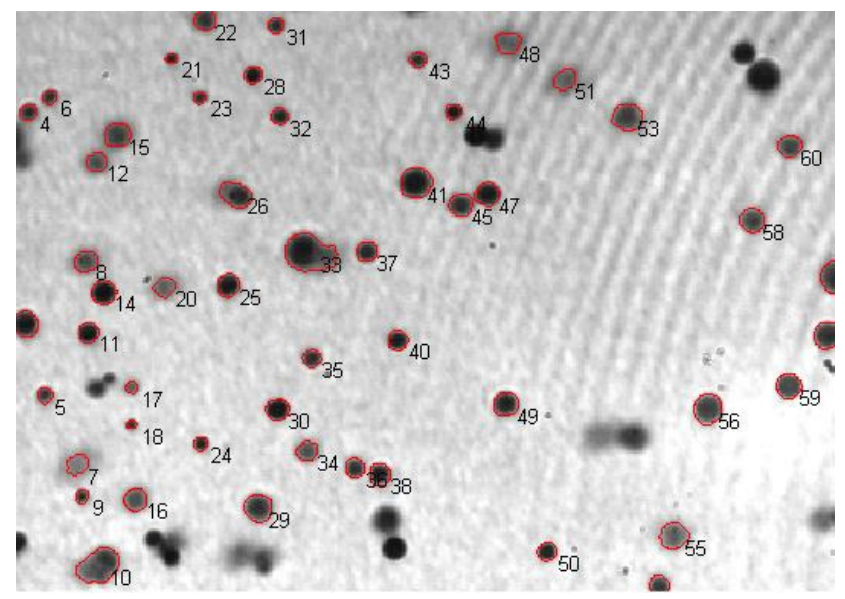

Figure 7. Contours of qualified droplets (with red ring and a number) and an index number superimposed on $\boldsymbol{I}_{\boldsymbol{i}}$.

\subsection{Droplet matching}

To find the velocity of the droplets, the position of each droplet in a given frame is compared with the position of the same droplet in subsequent frames. In this article, the velocity of the droplets in image $T_{i}$ is calculated with the use of three images, i.e. $T_{i-1}, T_{i}$ and $T_{i+1}$. The velocity distribution of the droplets is then obtained by comparing the physical position in the temporally adjacent frames.

Therefore, to calculate the droplet velocity, all "infocus" droplets have to be tracked between the frames. However, this is a challenging problem, since several possible droplet paths may exists for a given set of droplets, see Figure 8. By using brute-force search, the number of possible configurations of droplet paths for $n$ droplets are $n$ !. By using the Hungarian algorithm the number will only be $n^{2}$.

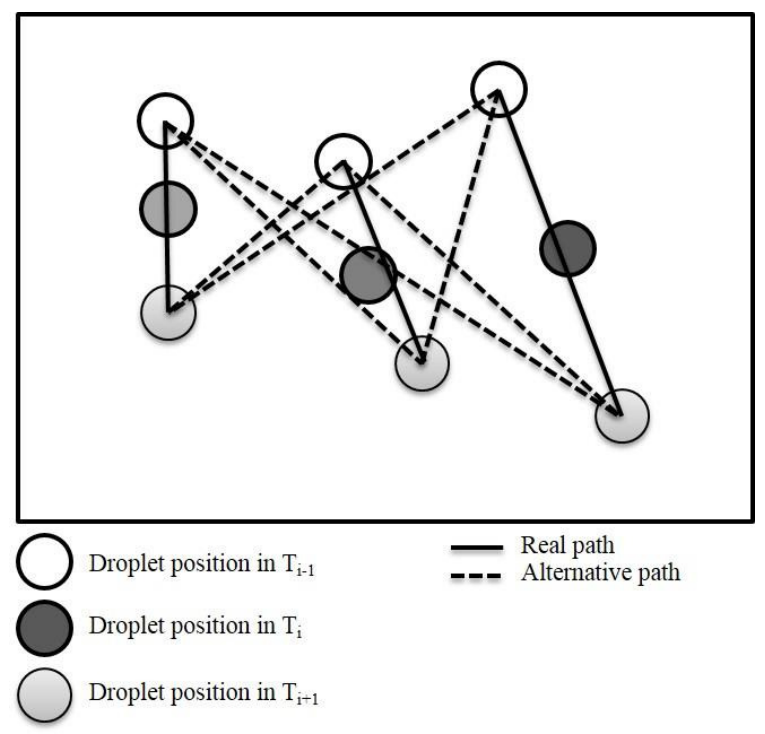

Figure 8. Droplet combinations for illustrations. Possible paths for three droplets in three sub sequential frames.

Please note that, with respect to the flow properties, each droplet has exactly one path, i.e. one single droplet cannot be assigned to several paths. Moreover, the number of "in-focus" droplets may change from frame to frame, as droplets might emerge or disappear in subsequent frames. To overcome these challenges, a multiple tracking framework is applied. Multiple tracking considers all possible paths, and selects the optimal combination. Such an optimization problem can be solved efficiently by, e.g. the Hungarian algorithm (Kuhn, 1955).

\subsection{Tracking as an assignment problem}

In terms of mathematics, a path between the positions of droplets in subsequent frames can be assigned a cost. In this thesis, the cost for a given path is defined by a combination for the following constraints: 
- The direction of the path should not change appreciable from frame to frame.

- The droplet velocity should not deviate much from an average droplet velocity.

- The droplet size should not differ appreciably from frame to frame.

- A droplet cannot be assigned to more than one path.

To find the optimal association of all the droplets in adjacent frames, a cost must be calculated for $\boldsymbol{e a c h}$ individual path. Let $P=\left\{p_{1}, p_{2, \ldots}, p_{k}\right\}, \quad Q=$ $\left\{q_{1}, q_{2, \ldots,} q_{l}\right\}$ and $R=\left\{r_{1}, r_{2, \ldots,} r_{m}\right\}$ denote the sets of $k, l$ and $m$ points (droplets) detected in frame $i-1, i$ and $i+1$, respectively. The total cost of each path from $p \in P$ to $r \in R$ via $q \in Q$ is given by the penalty matrix $C_{k \times m}$, where

$$
C_{k \times m}=\delta \Delta_{k \times m}+\lambda \Lambda_{k \times m}+\gamma \Gamma_{k \times m}
$$

and $\delta, \lambda$ and $\gamma$ are positive constants and the subscript $k \times m$ represents the dimension of the matrixes, i.e. the matrixes have $k$ rows and $m$ columns. In the following, the three matrixes $\Delta_{k \times m}, \Lambda_{k \times m}$ and $\Gamma_{k \times m}$ are discussed in more detail.

The elements in $\Delta_{k \times m}$ represents a measure of the smoothness of each path, and paths that change direction from one frame to another are penalized. To calculate the direction of a path, we utilize the fact that every convex combination of two points lies on the line segment between the two points, i.e. $w=\alpha p+$ $(1-\alpha) r$, with $0 \leq \alpha \leq 1$. By using $\alpha=1 / 2$, (assuming no acceleration), a point halfway between $p$ and $r$ is thereby simply given by $w=\frac{p+r}{2}$. If $w \in Q$, it means that there is a droplet in frame $i$ positioned halfway between a droplet $p \in P$ and a droplet $r \in R$, indicating a non-changing direction of the path. Otherwise, with $w \notin Q$, the path changes direction from one frame to another, and the changes are measured as the shortest distance from $w$ to any $q \in Q$, i.e.

$$
\Delta_{k \times m}=\left[\begin{array}{ccc}
d\left(\frac{p_{1}+r_{1}}{2}, q^{*}\right) & \cdots & d\left(\frac{p_{1}+r_{m}}{2}, q^{*}\right) \\
\vdots & \ddots & \vdots \\
d\left(\frac{p_{k}+r_{1}}{2}, q^{*}\right) & \cdots & d\left(\frac{p_{k}+r_{m}}{2}, q^{*}\right)
\end{array}\right]
$$

where $d\left(w, q^{*}\right)$ denotes the shortest distance from $w$ to any $q \in Q$.

All droplets will move a certain distance between the frames in the high speed movie. However, the droplet velocity depends on the droplet size, i.e. larger droplets move with another velocity than smaller droplets. Each element in $\Lambda_{k \times m}$ in (3) gives the deviation between the velocity along a given path and a size dependent (priori calculated) velocity distribution by

$$
\Lambda_{k \times m}=\left[\begin{array}{ccc}
\left|d\left(p_{1}, r_{1}\right)-v^{*}\right| & \cdots & \mid d\left(p_{1}, r_{m}\right)-v^{*} \\
\vdots & \ddots & \vdots \\
\left|d\left(p_{k}, r_{1}\right)-v^{*}\right| & \cdots & \mid d\left(p_{k}, r_{m}\right)-v^{*}
\end{array}\right.
$$

where $\left|d(p, r)-v^{*}\right|$ denotes the absolute value of the distance (velocity) by moving from $p$ to $r$ (the framerate is known), minus a size dependent velocity distribution, where the size dependent velocity $v^{*}$ is based on droplet $p$ and $r$.

Finally, the elements in $\Gamma_{k \times m}$ are a measure of the mismatch in the size of the droplets connected along a given path. First, let us introduce the following notation

$$
A(p, q, r)=\frac{|A(p)-A(q)|+|A(p)-A(r)|+|A(q)-A(r)|}{A(p)+A(q)+A(r)} .
$$

where $A(p), A(q)$ and $A(r)$ denotes the area of droplets $p, q$ and $r$, respectively. With this notation at hand, the matrix $\Gamma_{k \times m}$ is given by

$$
\Gamma_{k \times m}=\left[\begin{array}{ccc}
A\left(p_{1}, q^{*}, r_{1}\right) & \cdots & A\left(p_{1}, q^{*}, r_{m}\right) \\
\vdots & \ddots & \vdots \\
A\left(p_{k}, q^{*}, r_{1}\right) & \cdots & A\left(p_{k}, q^{*}, r_{m}\right)
\end{array}\right]
$$

where $q^{*}$ is the droplet $q \in Q$ with the shortest distance to the midpoint between the given $p$ and $r$.

Please note that all elements in the matrixes $\Delta_{k \times m}$, $\Lambda_{k \times m}$ and $\Gamma_{k \times m}$ are greater or equal to zero. Consequently, all elements, i.e. costs, in the penalty matrix $C_{k \times m}$ are greater or equal to zero. The Hungarian algorithm takes the penalty matrix $C_{k \times m}$ as its only argument and returns the optimum path assignment (see (Kuhn, 1955) for details). The running time of the Hungarian is $O\left(n^{3}\right)$ compared to brute-force search of $O(n !)$.

Figure 9 shows the droplet paths with a red vector found with the Hungarian algorithm. The contours of the segmented droplets and the segmented droplets of the temporally adjacent frames are superimposed for illustrational purposes. 


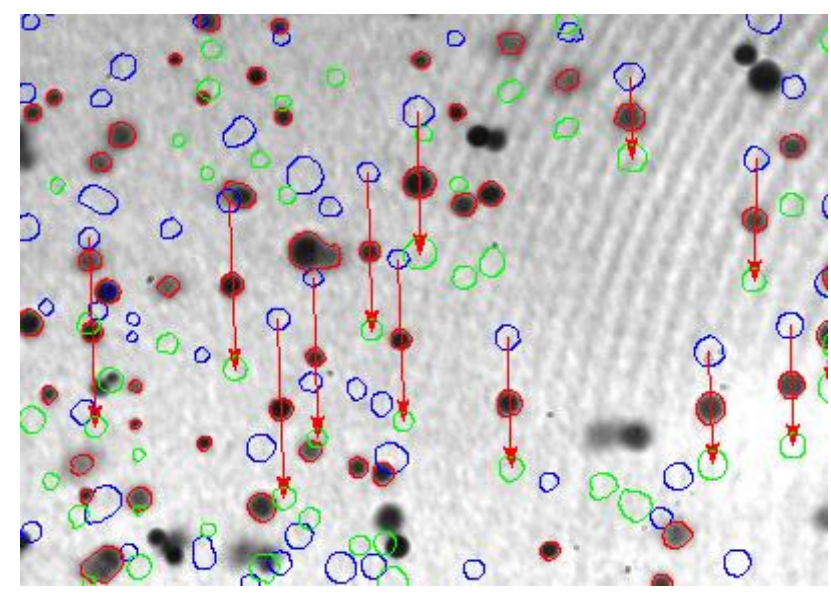

Figure 9. Vectors of droplet paths showing movement from frame $\boldsymbol{i}-\mathbf{1}$ (blue contour) to frame $\boldsymbol{i}+\mathbf{1}$ (green contour) of the droplets in frame $\boldsymbol{i}$ (red contour).

\section{Results}

This work gives preliminary results for one water pressure of $2.00 \mathrm{bar}(\mathrm{g})$ applied to the fire water nozzle. All the experiments are done one meter below the nozzle tip and at radial positions $0-150 \mathrm{~cm}$ from the center and at 3 different azimuthal angles $\left(0^{\circ}, 15^{\circ}\right.$ and $\left.30^{\circ}\right)$ compared to the frame arms of the nozzle.

Figure 10 shows the results of droplet size distribution when combining all the measurements from $0-150 \mathrm{~cm}$.

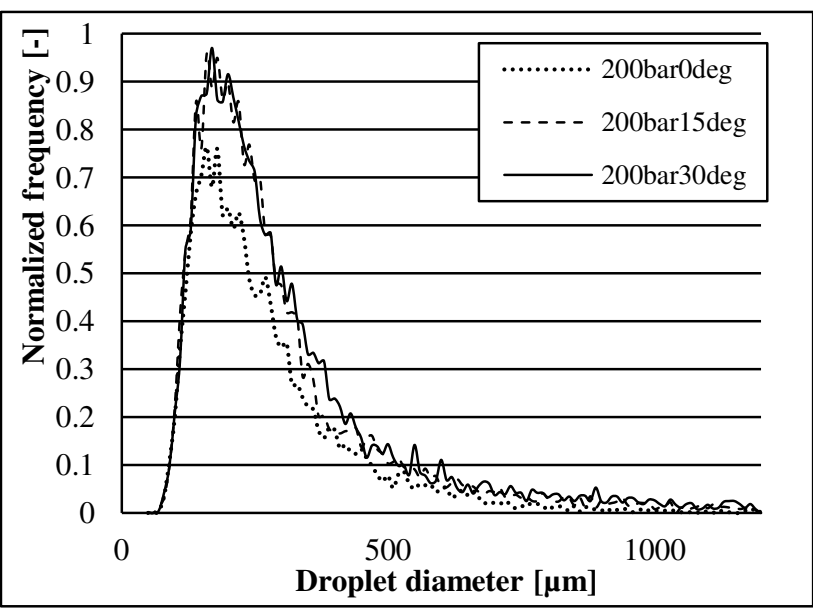

Figure 10. Total droplet size distribution for azimuthal angles of $\left(0^{\circ}, 15^{\circ}\right.$ and $\left.30^{\circ}\right)$ compared to the frame arms of the nozzle.

Figure 11 shows the velocity distribution in the spray. This is for all radial positions and the azimuthal angle $0^{\circ}$.

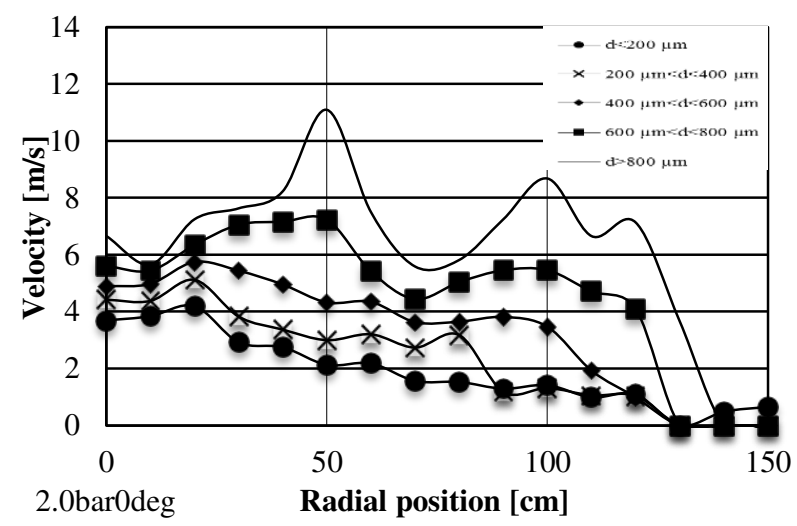

Figure 11. Preliminary experimental results for velocity of droplet size intervals as a function of radial position.

\section{Conclusion}

The usage of the Hungarian algorithm on a penalty matrix seems to give good results. The droplet paths, velocity and sizes seem to agree with spot checks by monitoring visually how the automatic image processing code perform on the images. The vectors in Figure 9 indicate that all the droplets flow in the same direction. This agrees with the physical properties of this spray. By using the velocity field of Figure 9 as an example, 12 droplet paths are found. To find these 12 paths with the Hungarian algorithm, a 12x12 matrix has to be solved. By using a brute-force search, almost 500 million possible path configurations have to be checked.

\section{References}

Gonzalez, R.C. and Woods, R.E. Digital Image Processing (third ed.). Pearson Prentice Hall, Upper Saddle River, NJ, 2008.

Jackman, L.A. Sprinkler Spray Interactions with Fire Gases. Ph.D. Thesis, Explosion and Fire Unit, South Bank University, London, 1992.

Kashdan, J.T., Shrimpton, J.S., Whybrew, A. A Digital Image Analysis Technique for Quantitative Characterisation of High-speed Sprays. Optics and Lasers in Engineering 45(1): 106-115, 2007.

Koh K.U., Kim J.Y., Lee S.Y. Determination of in-focus criteria and depth of field in image processing of spray particles. Atomization and Sprays, 11(4):317-333, 2001.

Kuhn, H.W. The Hungarian method for the assignment problem. Naval Research Logistics Quarterly, 2: 83-97, 1955.

Lecuona, A., Sosa, P.A., Rodriguez, P.A., Zequeira, R.I. Volumetric characterization of dispersed two-phase flows by digital image analysis. Measurement Science and Technology, 11: 1152-1161, 2000.

Lee S.Y., Kim Y.D. Sizing of Spray Particles Using Image Processing Technique. KSME International Journal, 18(6): 879-894, 2004.

Mathworks. MATLAB User's Guide (r2011b). 3 Apple Hill Drive Natick, MA, 2011. 
NORSOK-Standard, Technical Safety S-001 (4th ed). Standards Norway, 2008.

Otsu, N. A Threshold Selection Method from Gray-Level Histograms. IEEE Transactions on Systems, Man, and Cybernetics 9(1): 62-66, 1979.

Sheppard, D.T. Spray Characteristics of Fire Sprinklers. National Institute of Standards and Reporting NIST GCR 02-838, Gaithersburg, MD, 2002.

Zhou, X., D’Aniello, S.P., Yu, H.-Z. Spray Characterization Measurements of a Pendent Fire Sprinkler. Fire Safety Journal 54: 36-48, 2012. 